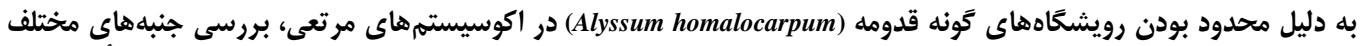

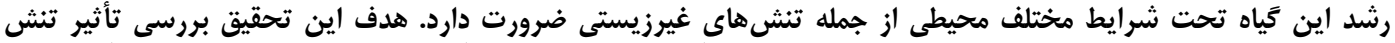

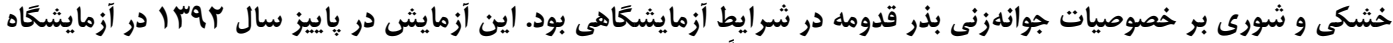

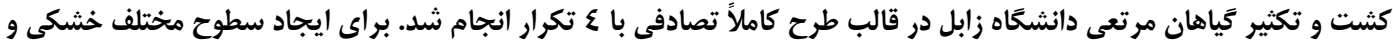

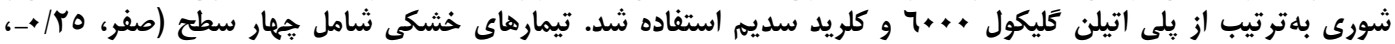

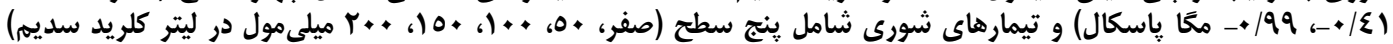

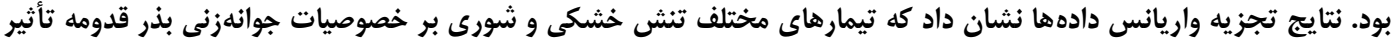

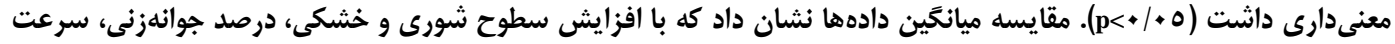

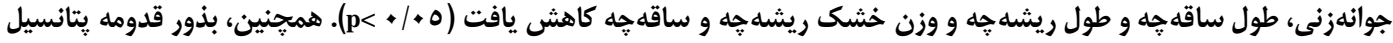

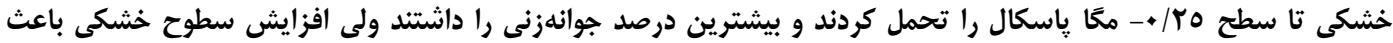

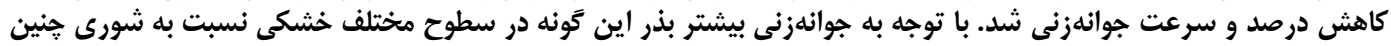

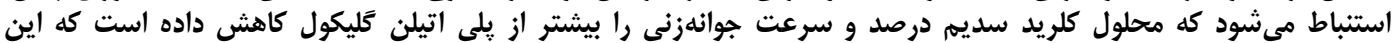

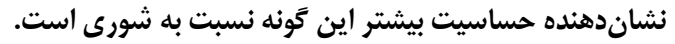

وازههاى كليدى: تنش خشكى، تنش شورى، جوانهزنى، قدومه، كَياه دارويى

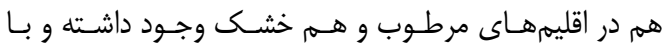

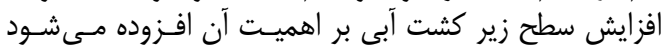

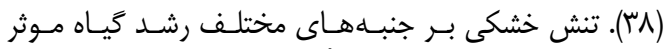

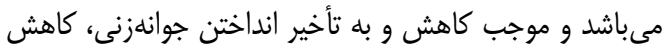

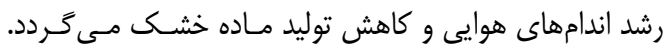

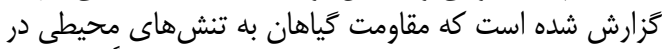

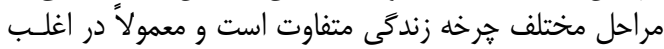

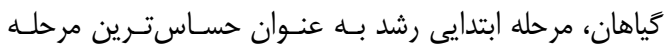

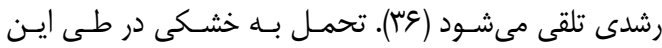

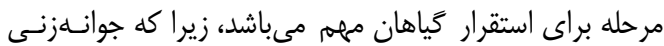

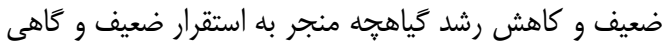

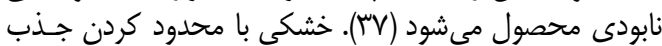

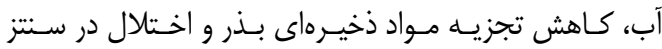

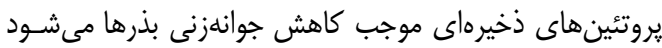

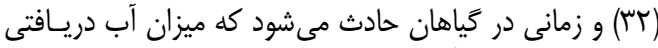

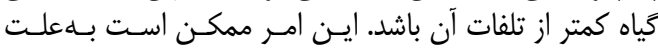

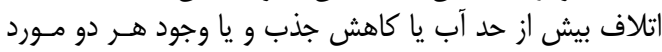

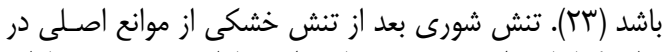

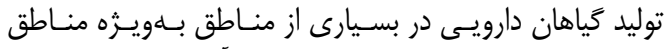

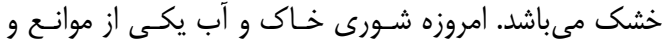

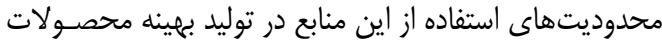

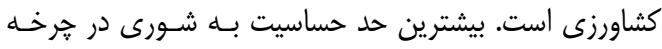

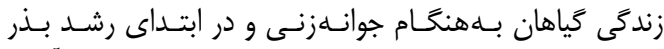

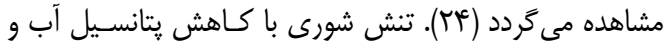

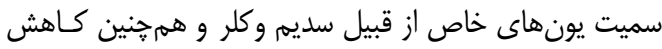

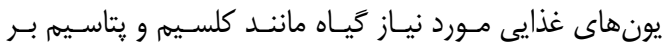

مقدمه

شناسائى، اهلـى سـازى و مطالعـه اصـول كاشـت كياهـان

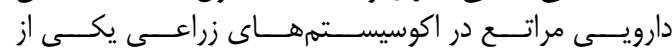

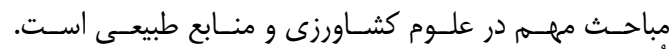

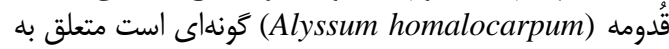

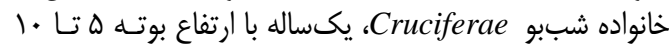

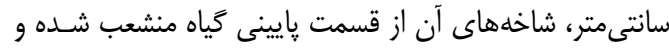

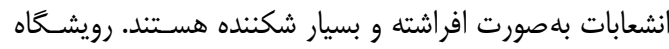

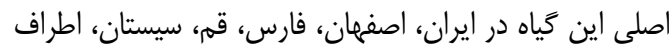

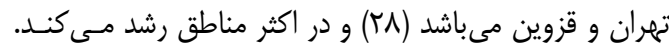

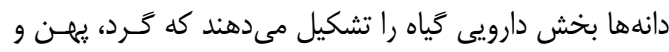

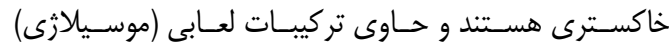

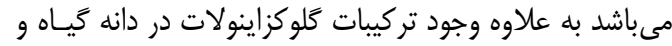

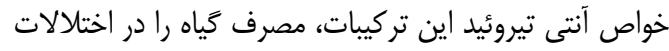

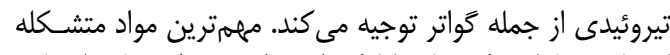

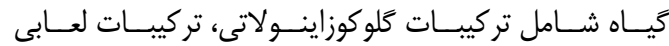

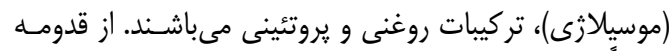

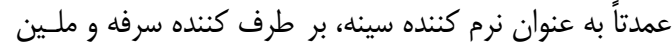

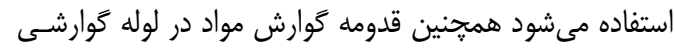

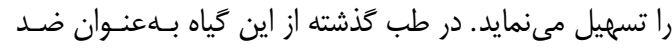

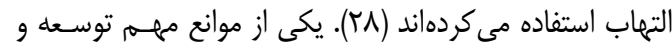

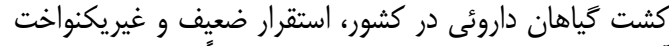

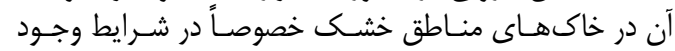

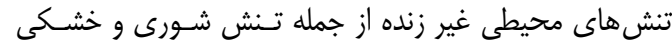

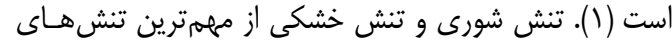

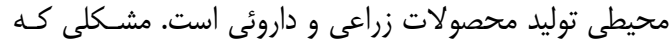




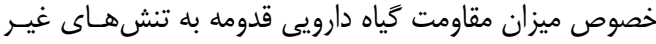

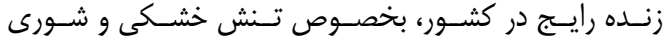

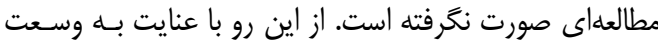

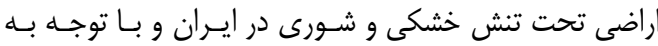

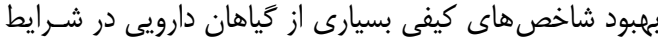

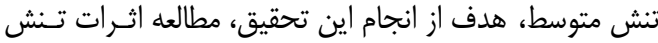

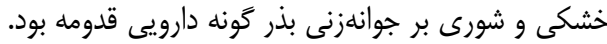

\section{مواد و روشها}

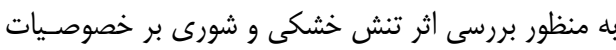

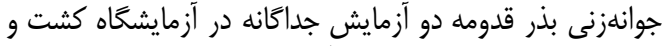

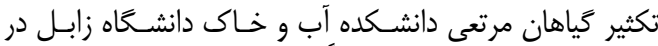

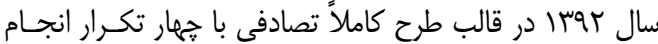

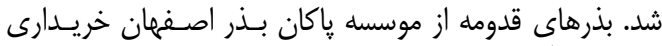

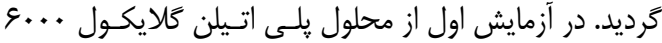

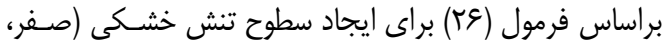

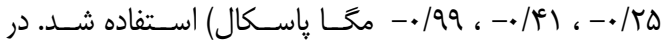

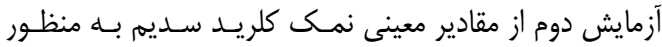

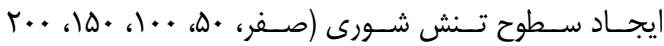

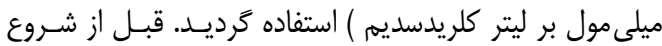

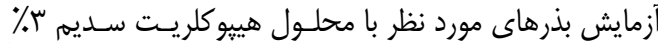

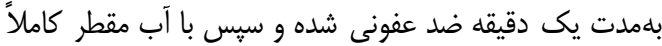

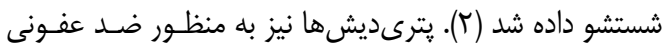

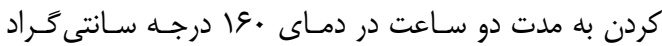

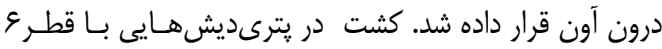

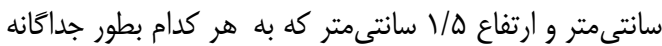

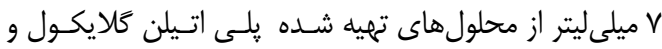

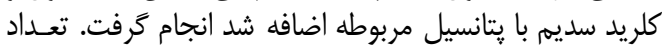

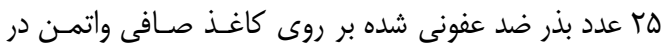

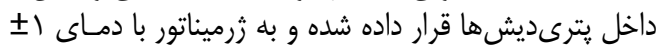

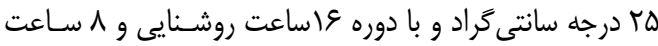

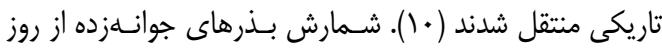

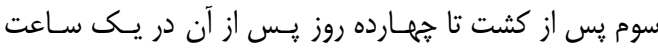

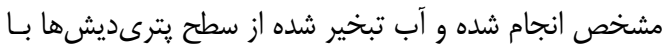

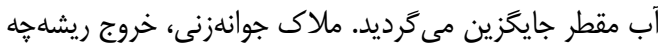

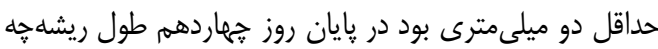

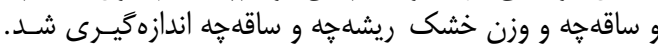

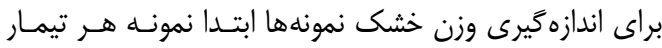

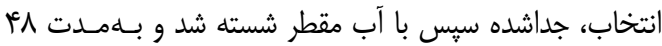

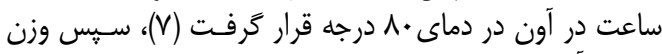

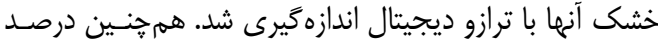

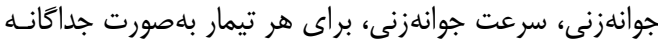

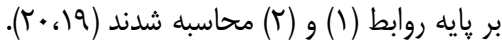
$G P=(N i / S) \times 100$

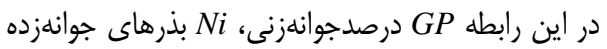
در هر روز و S تعدادكل بذر مىباشد.

$G R=\sum n i / t i$

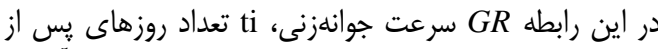

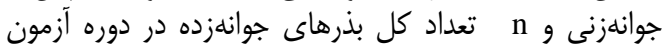

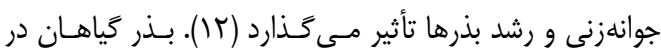

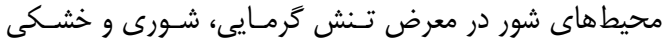

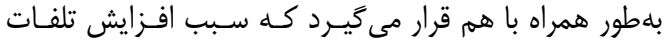

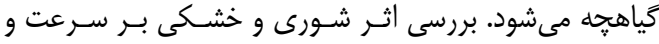

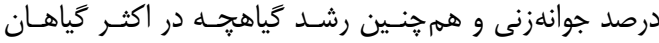

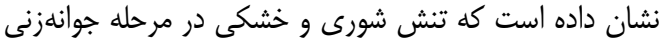

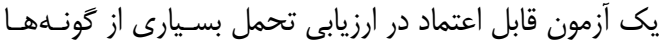

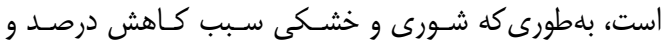

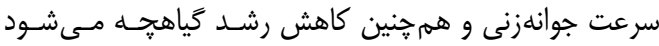

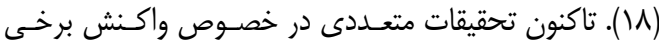

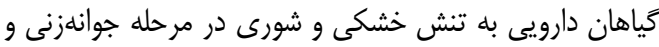

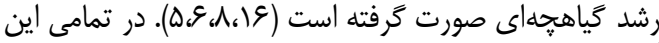

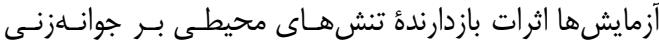

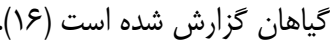

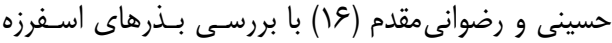

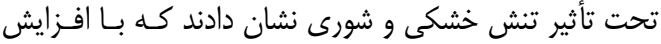

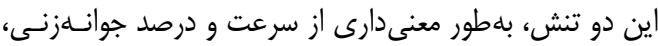

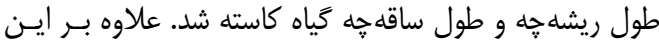

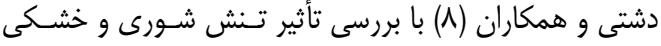

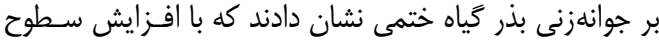

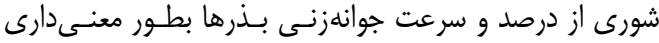

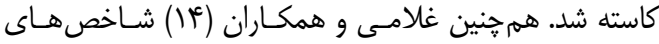

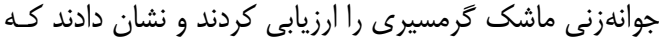

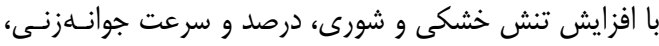

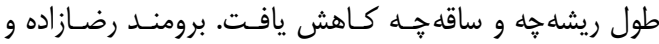

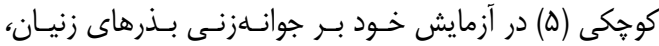

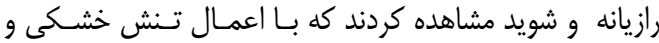

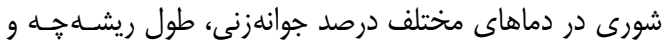

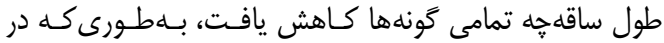

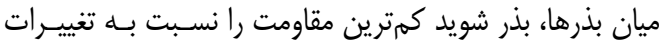

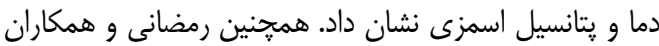

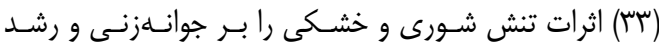

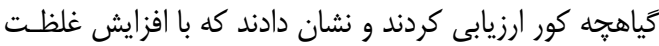

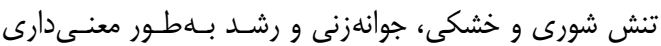

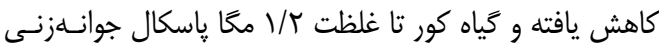

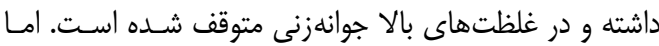

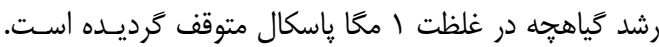

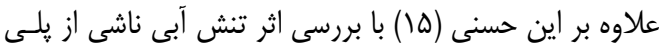

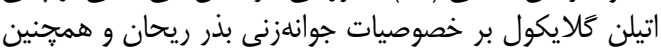

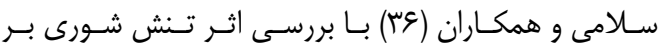

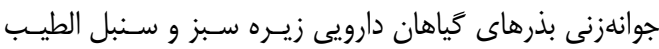

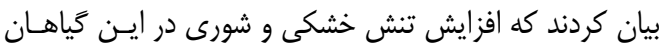

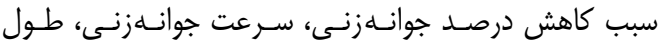

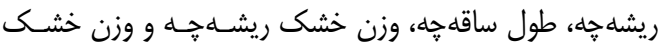

ساقه קه مى شود.

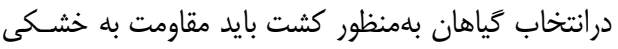

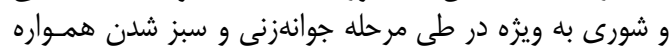

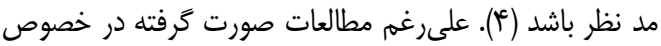

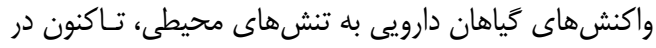




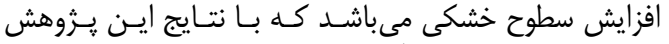

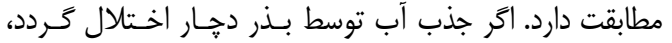

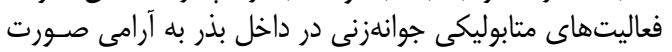

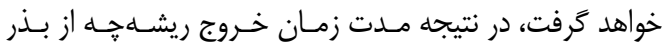

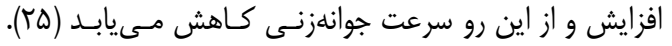

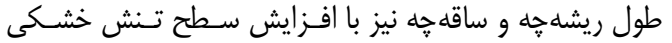

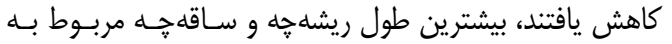

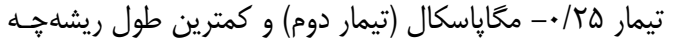

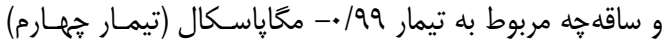

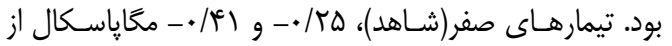

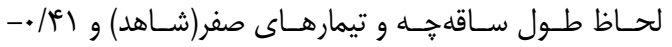

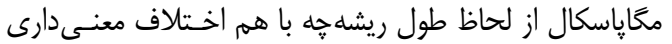

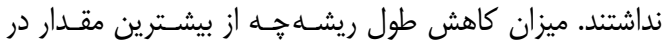

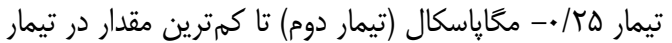

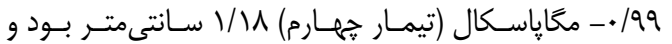

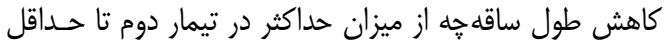

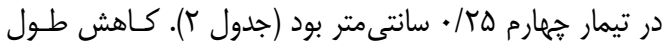

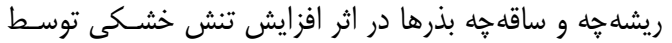

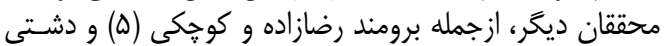

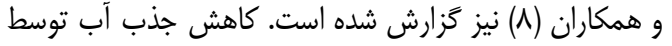

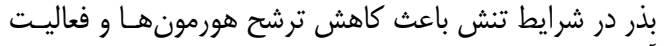

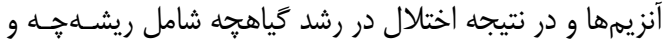

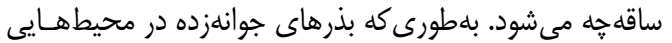

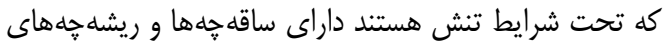

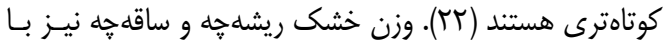

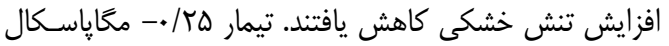

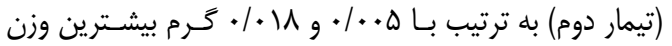

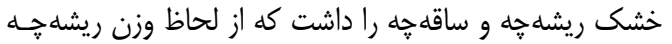

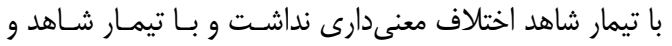

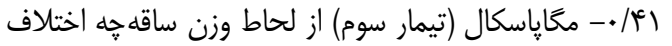

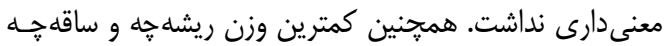

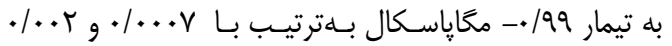

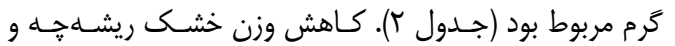

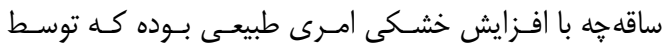

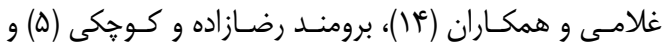

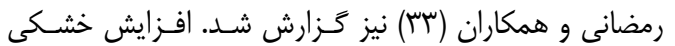

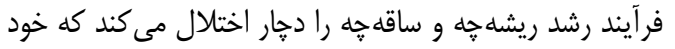

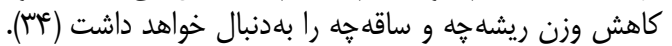

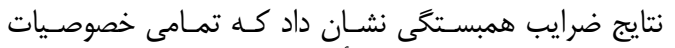

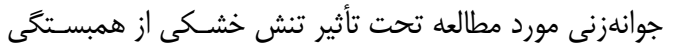

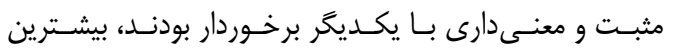

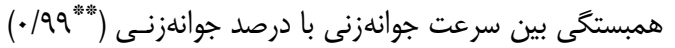

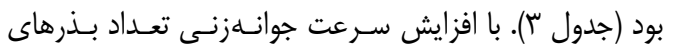

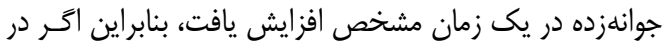

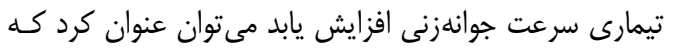

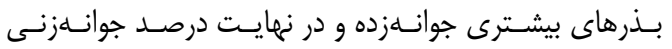

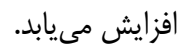

مىباشد. در اين آزمايش براى تجزيه و تحليل دادهها از داز

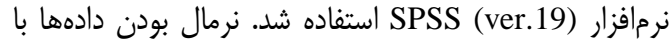

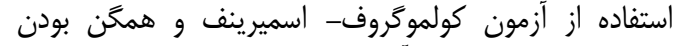

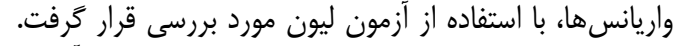

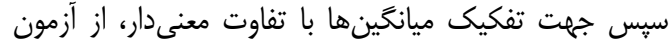

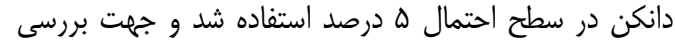

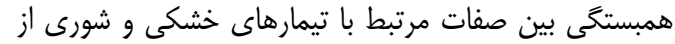
آزمون ضريب همبستخى ييرسون استفاده شد.

\section{نتايج تجزيه واريـانس دادههـا، نشـان داد كـهـ تيمارهـاى}

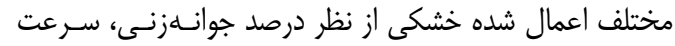

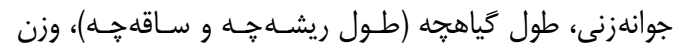

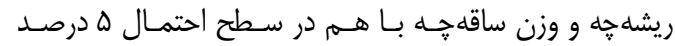

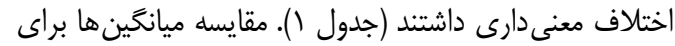

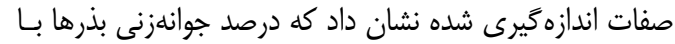

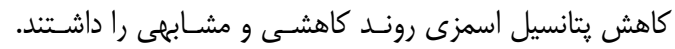

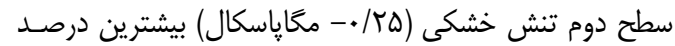

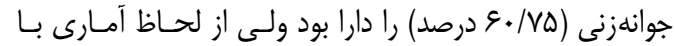

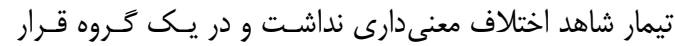

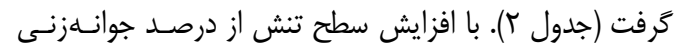

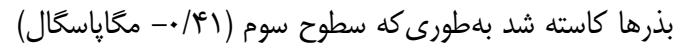

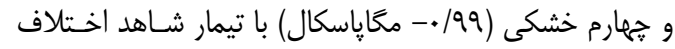

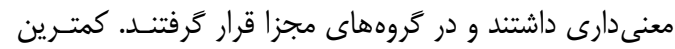

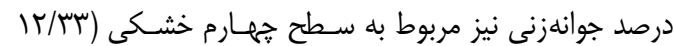

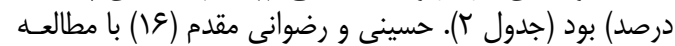

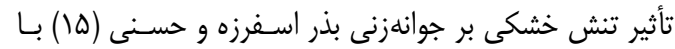

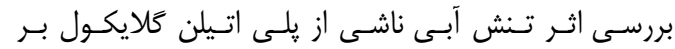

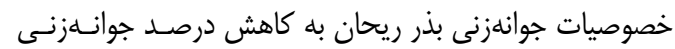

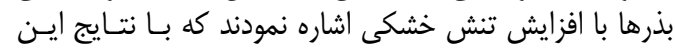

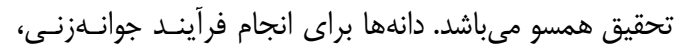

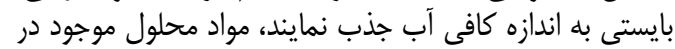

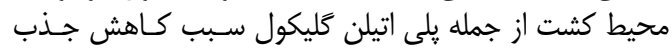

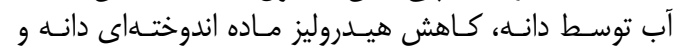

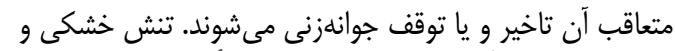

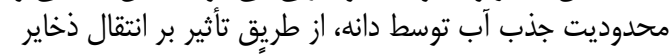

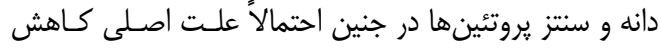

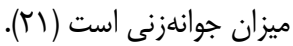

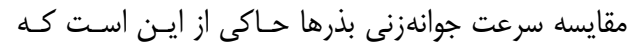

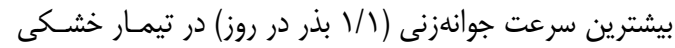

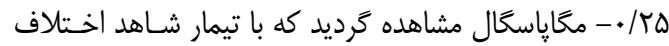

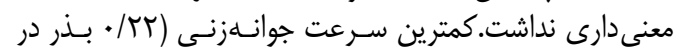

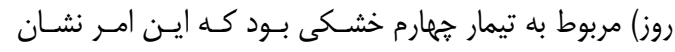

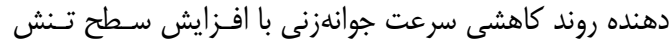

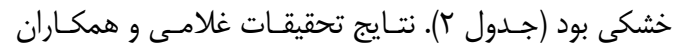

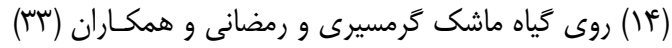

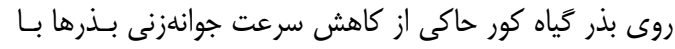



تأثير تنشهاى خشكى و شورى بر جوانهزنى بذر گياه قدومه

جدول 1 - تجزيه واريانس (ميانخين مربعات) خصوصيات جوانهزنى بذر قدومه در سطوح مختلف خشكى (ئلى Table 1. Analysis of variance (mean square) at different levels of drought on seed germination characteristics of Allsuysm

\begin{tabular}{|c|c|c|c|c|c|c|c|}
\hline ريشه & ساقه קه & ريشه & ساقهُ & جوانهزنى & درصد جوانهزنى & آدرادى & منابع تغييرات \\
\hline$\cdot / \cdots \Delta \Lambda^{*}$ & $\cdot 1 \ldots 9^{*}$ & $T / V V^{*}$ & $\cdot / \mathrm{N}^{*}$ & $1 / 9^{*}$ & $\Delta V \cdot 9 / 4)^{*}$ & $r$ & تيمار \\
\hline ג & $.1 \cdots \Delta$ & .119 & $.1 . \Delta V$ &.$/ 19$ & $\Delta \Delta T / V V$ & $\pi$ & خطا \\
\hline
\end{tabular}

جدول r- مقايسه ميانگين خصوصيات جوانهزنى بذر گياه قدومه تحت تأثير سطوح مختلف خشكى Table 2. The average of germination characteristics of Allyssum under different drought treatments

\begin{tabular}{|c|c|c|c|c|c|c|}
\hline وزن خشك ساقهاهِ & وزن خشك ريشه جه & $\begin{array}{l}\text { طول ريشه } \\
\text { (cm) }\end{array}$ & 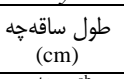 & 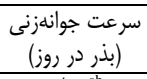 & درصد جوانهزنى & منبع تغييرات \\
\hline.$/ \cdot 19^{a}$ &.$/$. cab $^{\text {a }}$ & $1 / / r^{\mathrm{a}}$ & $. / 99)^{\mathrm{ab}}$ & $\cdot / 1 q^{a b}$ & $\Delta \Psi / \Psi^{a^{a^{*}}}$ & شاهد (صفر) \\
\hline$\cdot / \cdot 1 \Lambda^{\mathrm{a}}$ & $\cdot / \cdot \Delta^{\mathrm{a}}$ & $1 / v \omega^{b}$ & $\cdot|\Lambda| r^{a}$ & $1 / 1 \cdot^{a}$ & $G \cdot / V Q^{a}$ & ه//.- مخاياسكال \\
\hline.$/ .11^{\mathrm{ab}}$ & $\cdot / . . p^{b c}$ & $1 / 1 \omega^{a}$ & . $199 \mathrm{r}^{\mathrm{ab}}$ & $\cdot / \Delta \Lambda^{b}$ & $r Y / \mathscr{G} V^{b}$ & أ|+.- مخائاسكال \\
\hline.$\cdot \cdot r^{\mathrm{D}}$ & $\cdot / \cdots \gamma^{c}$ & $\cdot / \Delta \gamma^{c}$ & $\cdot / \Delta \Delta \Delta^{D}$ & $\cdot / r^{\mathrm{c}}$ & $\mid r / \Psi^{c}$ & 99/•- مخإِاسكال \\
\hline
\end{tabular}

جدول r- مقادير ضرايب همبستخى (ييرسون) بين صفات مرتبط با سطوح مختلف خشكى در گياه قدومه Table 3. The correlation coefficient s (Pearson) related to different levels of drought in Allyssum

\begin{tabular}{|c|c|c|c|c|c|c|}
\hline وزن خشك ريشه קه & وزن خشك ساقه هִ & طول ريشه & طول ساقه خه & سرعت جوانهزنى & درصد جوانهزنى & خصوصيات جوانهزنى \\
\hline & & & & & 1 & درصد جوانهزنى \\
\hline & & & & 1 & . $/ 99^{\text {*atus }}$ & سرعت جوانهزنى \\
\hline & & & 1 & $\cdot \mid \Delta \Lambda^{*}$ & $.109^{*}$ & طول ساقهجه \\
\hline & & 1 & 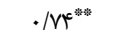 & $\cdot / N Y^{* * w}$ & $\cdot / V \varphi^{* * * w}$ & طول ريشه \\
\hline & 1 & $\cdot 19 \Lambda^{* 0 *}$ & $\cdot \mid \Delta F^{*}$ & 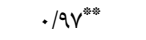 & 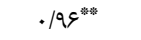 & وزن ساقه ֶه \\
\hline 1 & $\cdot / 19^{* * *}$ & $\cdot / N N^{* *}$ & $\cdot / \Delta V^{\circ}$ &.$/ 99^{* * * a s}$ & $\cdot / 90^{* t * a s}$ & وزن ريشه \\
\hline
\end{tabular}

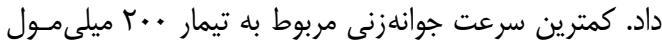

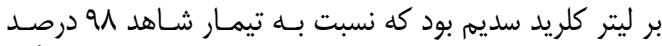

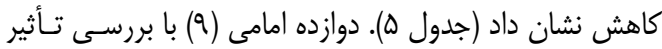

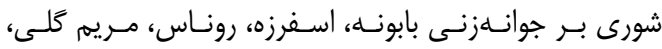

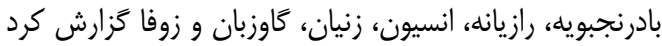

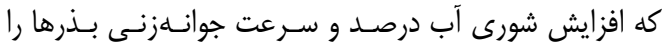

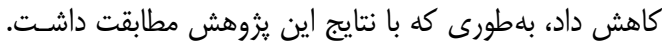

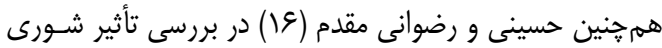

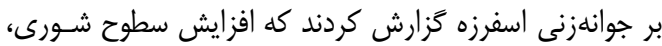

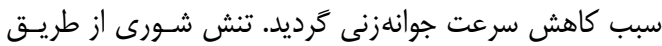

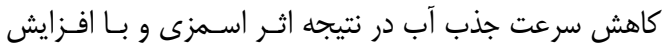

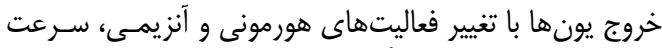

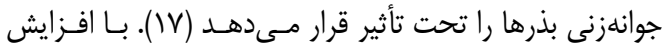

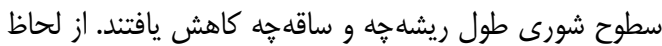

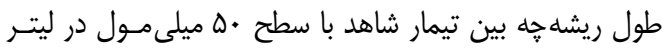

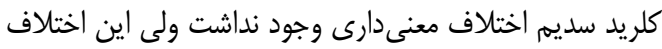

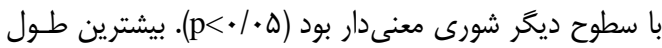

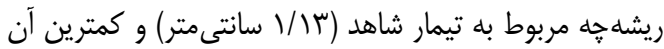

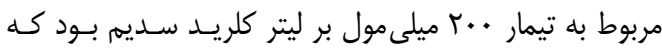

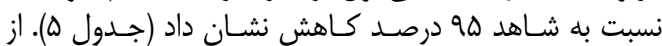

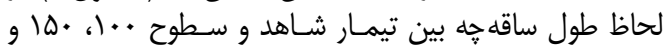

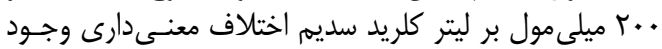

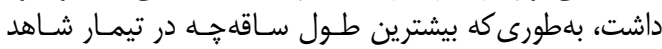

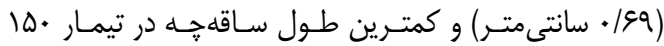

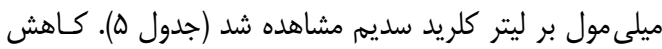

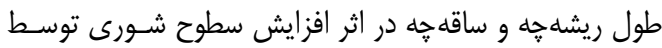

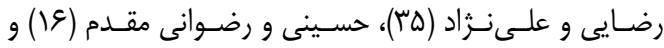

تنث شورى

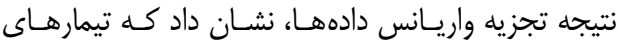

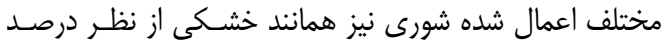

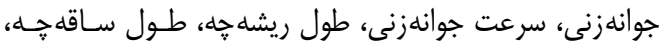

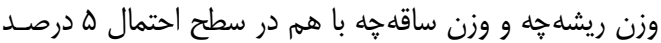

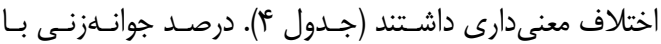

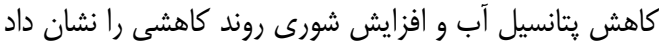

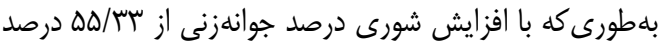

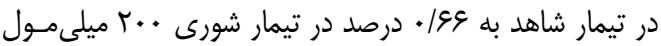

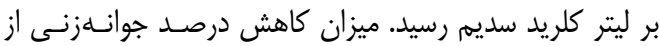

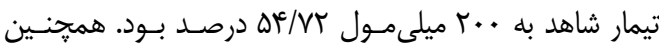

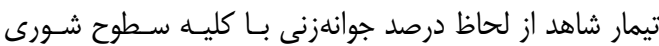

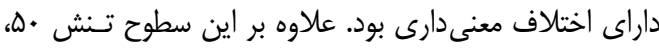

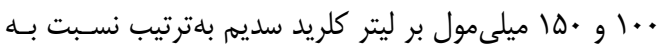

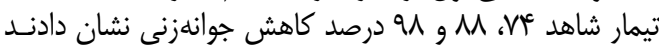

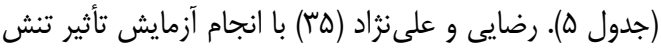

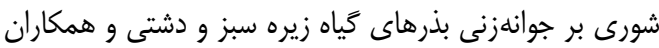

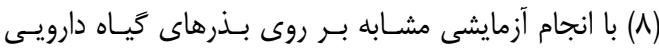

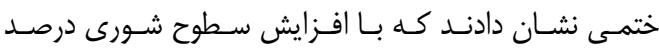

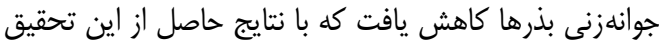

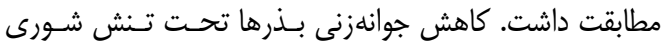

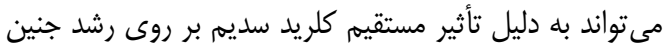

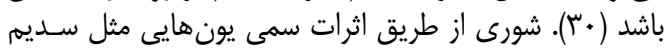

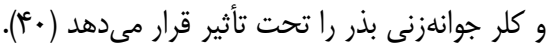

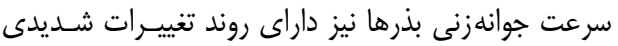

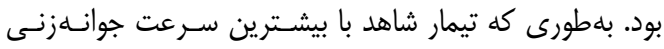

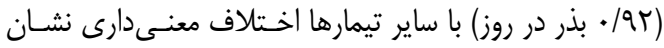


لحاظ وزن خشك ساقه جه بـين تيمـار شـاهد و كليـه سـطوح

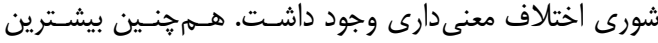

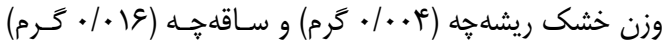

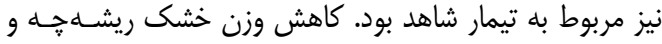

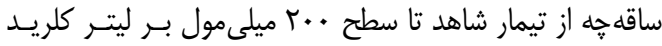

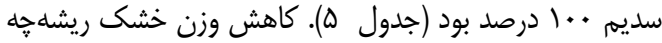

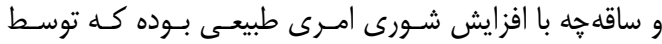

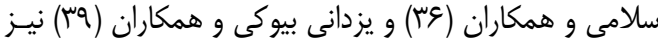

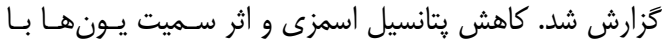

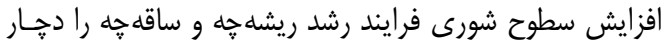

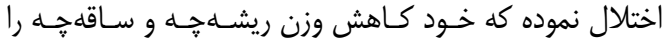

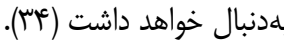

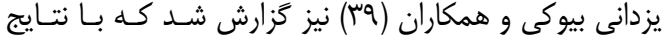

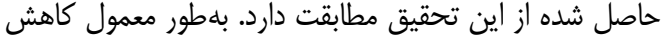

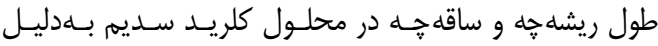

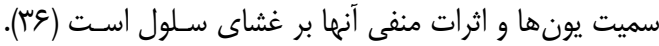

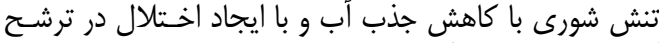

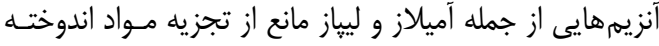

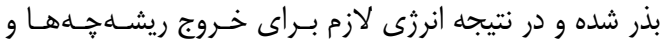

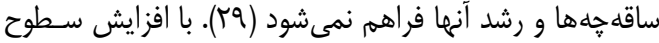

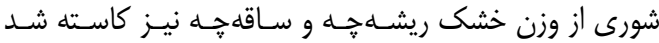

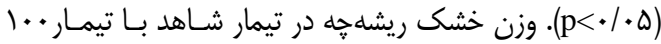

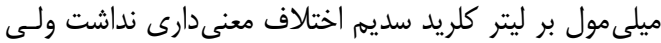

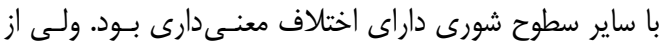

جدول f- تجزيه واريانس (ميانكَين مربعات) خصوصيات جوانهزنى بذر قدومه در سطوح مختلف شورى (لمى Table 4. Analysis of variance (mean square) at different levels of salinity on seed germination characteristics of Allyssum

\begin{tabular}{|c|c|c|c|c|c|c|c|}
\hline وزن ساقه هِ & وزن ريشهاهه & طول ساقه & طول ريشه & سرعت جوانهزنى & درصد جوانهزنى & درجه آزادى & منابع تغييرات \\
\hline$\cdot / \cdots 1^{3}$ & $\cdot / \cdots \Delta \Lambda^{* \prime \prime}$ & $1 / 1$. & r/Ir" & $1 / 94^{* 3}$ & $\Delta \wedge \backslash \varepsilon / \ldots$ & r & تيمار \\
\hline$/ \ldots r$ & $\% \ldots r$ & .1 .9 &.$/ 11$ &.$/$. & $r \cdots / \wedge q$ & 10 & خطا \\
\hline
\end{tabular}

جدول ه- مقايسه ميانگَين خصوصيات جوانهزنى بذر گَياه قدومه تحت تأثير سطوح مختلف شورى Table 5. The average of germination characteristics of Allyssum under different salinity treatments

\begin{tabular}{|c|c|c|c|c|c|c|}
\hline ساقه خه (َرم) & ريشه خه (كرم) & 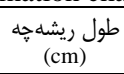 & 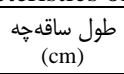 & 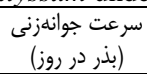 & درصد جوانهزنى & منبع تغييرات \\
\hline$\cdot / \cdot 19^{\mathrm{a}}$ & $\cdot / \cdot \varphi^{\text {ca }}$ & $1 / 1 r^{\mathrm{a}}$ &.$/ 99^{a}$ & $\cdot / 9 \mathrm{r}^{\mathrm{a}}$ & $\Delta \mathrm{Q} / \mathrm{rr}^{\mathrm{a} \mathrm{a}^{*}}$ & شاهد (صفر) \\
\hline$\cdot / \cdot \Delta^{\mathrm{b}}$ & $\cdot(\cdot . \cdot)^{\mathrm{b}}$ &.$/ 9 \Delta^{\mathrm{a}}$ & $\cdot / \Delta q^{a}$ & 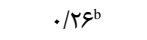 & $\mid x / 99^{b}$ & . ه ميلىمول بر ليتر كلريد سديم \\
\hline$\cdot / \cdot r^{b}$ & $\cdot / . . r^{\mathrm{ab}}$ &.$/ q^{b}$ &.$/ 19^{b}$ & $\cdot / / r^{b}$ & $V / r^{\mathrm{b}}$ & . . ميلىمول بر ليتر كلريدسديم \\
\hline.$/{ }^{\mathrm{b}}$ &.$/ \ldots^{\mathrm{b}}$ & $\cdot / \cdot 0^{\mathrm{b}}$ & $\cdot 1 \cdot r^{\mathrm{cb}}$ &.$/ \cdot 1 r^{b}$ & $.199^{b}$ & •ا ا ميلىمول بر ليتر كلريد سديم \\
\hline.$/ \ldots \mathrm{b}$ &.$/ \omega^{\mathrm{b}}$ & $\cdot 1 \cdot \Delta^{\mathrm{b}}$ & $.1 .9^{b}$ & $.1 \cdot 11^{\mathrm{b}}$ & $\cdot|q|^{\mathrm{b}}$ & • . ميلى مول بر ليتر كلريد سديم \\
\hline
\end{tabular}

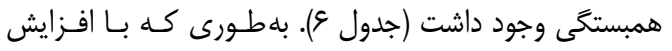

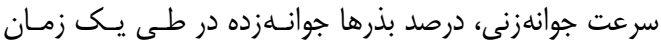
مشخص افزايش يافت.
نتايج حاصل از ضرايب همبستخى بين صـفات نشـان داد

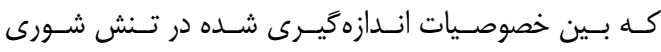

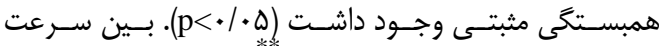

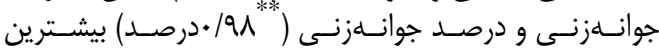

جدول \&- مقادير ضرايب همبستخى (ييرسون) بين صفات مرتبط با سطوح مختلف شورى در گياه قدومه Table 6. The correlation coefficient s (Pearson) related to different levels of salinity in Allyssum

\begin{tabular}{|c|c|c|c|c|c|c|}
\hline وزن خشك ريشه & وزن خشك ساقه & طول ريشه & طول ساقه & سرعت جوانهزنى & درصد جوانهزنى & خصوصيات جوانهزنى \\
\hline & & & & & 1 & درصد جوانهزنى \\
\hline & & & & 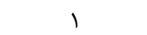 & $.19 \Lambda^{* * 3}$ & سرعت جوانهزنى \\
\hline & & & 1 & $\cdot 1 \Delta\rangle^{* * \omega *}$ & $\cdot \mid \Delta \Lambda^{* * \omega *}$ & طول ساقه \\
\hline & & 1 & 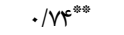 & $\cdot / V Y^{* * * 4}$ & $\cdot / V \omega^{* * *}$ & طول ريشهِه \\
\hline & 1 & $\cdot / N^{* * s}$ & $\cdot \mid \Delta \mu^{* \prime \prime}$ & $\cdot / v v^{* * *}$ & $\cdot / \mathrm{N}^{* * * *}$ & وزن خشك ساقه \\
\hline 1 & $\cdot / 19^{* * a}$ & $\cdot / \mathrm{V} \cdot{ }^{* * *}$ & $\cdot \mid \Delta \gamma^{*}$ & $\cdot / \mathrm{v}^{* * a}$ & $\cdot / 10^{* * * n}$ & وزن خشك ريشهٍه \\
\hline
\end{tabular}

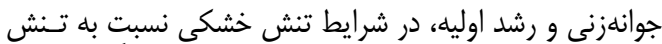

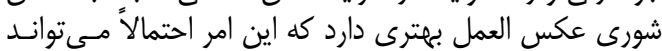

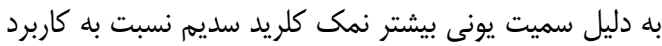

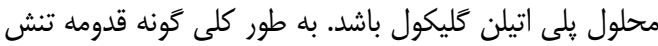

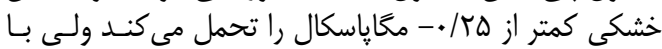

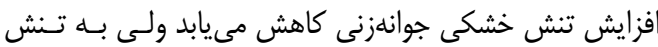

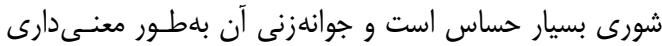

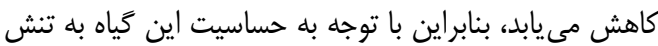

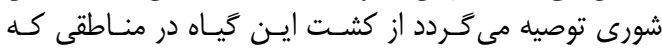
شورى خاك زياد است خوددارى شود.

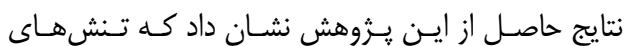

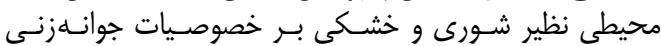

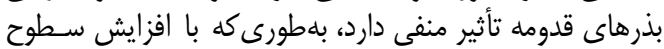

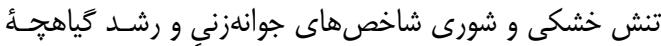

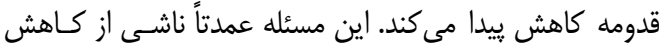

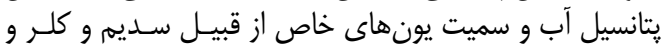

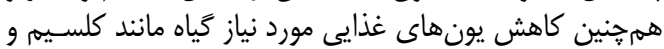

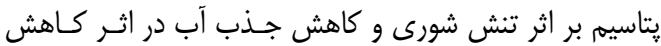

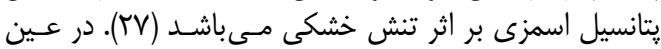

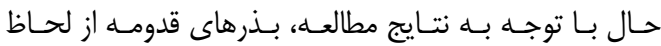


1. Alizadeh, A. 2002. Agricultural drought management. Journal of drought, 3: 3-7 (In Persian).

2. Aminifar, J., G. Mohsenabadi and S. Ghaderi. 2010. Effect of drought stress on germination and seedling growth of vetch (Vicia sp.). The First National Conference of Environmental stresses in agricultural science, University of Birjand, 28- 29 (In Persian).

3. Ashraf, M., H.R. Athar, P.J.C. Harris and T.R. Kwon. 2008. Some prospective strategies for improving crop salt tolerance. Advance Agronomy, 97: 45-110.

4. Bloom, A. and E.E. Pstein. 1984. Varietal differences in salt induced respiration in barely. Plant Physiology, 90: 1444-1456.

5. Boroumand Rezazadeh, Z. and A. Koocheki. 2005. Germination response of Ajowan,Fennel and Dill to osmotic potential of sodium chloride and polyethylene glycol 6000 in different temperature regimes. Iranian Journal of Field crops Research, 3: 207-217 (In Persian).

6. Burnett, S., P. Thomas and M. Van Iersel. 2005. Post germination drenches with PEG-8000 reduce growth of salvia and marigolds. Hort. Science, 40: 675-679.

7. Catalan, I., Z. Balzarini, E. Talesnik, R. Sereno and U. Karlin. 1994. Effect of salinity on germination and seedling growth of Prosopis flexuosa. Forest Ecology and Management, 63: 347-357.

8. Dashti, M., N. Sattar and E. Qurbanzadeh. 2007. Effect of drought and salinity stresses on germination of Althaea officinalis. First National Conference on Medicinal Plants and Sustainable Agriculture, $259 \mathrm{pp}$ (In Persian).

9. Davazdah-Emami, S. 2002. Effect of salinity and chilling on germination characteristics of medicinal chamomile. $7^{\text {th }}$ Iranian agronomy and plant breeding congress, 571pp (In Persian).

10. Draper, S.R., L.N. Bass, A. Bould, P. Gouling, M.C. Hutin, W.J. Rennie, A.M. Steiner and J.H.B. danTonkin. 1985. International Seed Testing Associtaion. Seed Science and Technology, 13 pp.

11. Fougere, F., D.L. Rudulier and J.G. Streeter. 1991. Effects of salt stress on amino acid, organic acid, and carbohydrate composition of roots bacteroids, and cytosol of alfalfa (Medicago sativa). Plant Physiology, 96: 1228-1236.

12. Ghaderi, S., J. Ghorbani, P. Gholami, A. Karimzadeh and F. Salarian. 2011. Effect of drought and salinity stresses on germination indices of vetch (vida villosa l.). Journal of Agroecology, 3: 120-129 (In Persian).

13. Ghanavati, M., S. Houshmand, H. Zainali and F. Ejlali. 2011. Salinity effects on germination and growth of chamomile genotypes. Journal of Medicinal Plants Research, 5: 6609-6614 (In Persian).

14. Gholami, P., J. Ghorbani, A. Karimzadeh, F. Salarian and S. Ghaderi. 2009. Assessment of germination indices in Visia monantha under salinity stress. The first National Conference of Environmental Stresses in Agricultural Science. The University of Birjand, 28: 464-471 (In Persian).

15. Hassani, A. 2005. Polyethylene glycol inducedwater stress on basil seed (Ocimum basilicum). Quarterly journal of Medicinal and Aromatic Plants Research of Iran, 4: 535-544 (In Persian).

16. Hosseini, H. and P. Rezvani Moghadam. 2006. Effect of water and salinity stress in seed germination on Isabgol (Plantago ovata). Iranian Journal Field Crop Research, 4: 15-22 (In Persian).

17. Huang, J. and R.E. Redmann. 1995. Salt tolerance of Hordeum and Brassica species during germination and early seedling growth. Canadian Journal Plant Science, 75: 59-81.

18. Irannejad, H., Z. Javanmardi, M. Golbash and M. Zarabi. 2009. Effect of drought stress on germination and early seedling growth in flax cultivars (Linum usitatissimum L.). 1st congress of oil crops. University of Isfahan, 154-156 (In Persian).

19. International Seed Testing Association (ISTA). 1995. Handbook of vigor test methods, Zurich, 117 pp.

20. International Seed Testing Association. 2008. International rules for seed testing. Seed Science and Technology, 24: 155-202.

21. Kaboli, M. and M. Sadeghi. 2001. Effect of drought stress on germination of three species of Onobrychis. Journal of Pajouhesh and Sazandegi, 64: 51-57 (In Persian).

22. Katergi, N., J.W. Van Hoorn, A. Hamdy, F. Karam and M. Mastrortilli. 1994. Effect of salinity on emergence andon water stress early seedling growth of sunflower and maize. Agricultural Water Management, 26: 81-91.

23. Koocheki, A.R. and A. Alizadeh. 1995. Agronomy with dry land. Astan Gods Razavi Publishers, Mashhad, Iran, $260 \mathrm{pp}$ (In Persian).

24. Maghtooli, M. and M.R. Chaeichi. 1999. Study of effect of salinity and salt type on germination and initial growth of Sorghum. Journal of Agricultural Sciences and Natural Resources of Gorgan, 4: 3340 (In Persian).

25. Mayer, A.M. and A. Polijakoff-Mayber. 1989. The Germination of Seeds. $4^{\text {ed }}$. Pergamon Press Oxford, Pergamon Press. Oxford, London, $270 \mathrm{pp.}$

26. Michel, B.E. and M.R. Kaufman. 1973. The osmotic potential of poly ethylene glycol 6000. Plant Physiology, 51: 914-916.

27. Misra, N. and U.N. Dwivedi. 1995. Carbohydrate metabolism during seed germination and seedling growth in green gram under saline stress. Plant Physiology, 33: 33-40.

28. Mozaffarian, V. 1996. Dictionary of Iranian Plant Names, Farhang Moaser, 2: 360-364 (In Persian).

29. Niu, X., R.A. Bressan, P.M. Hasegawa and J.M. Pardo. 1995. Ion homeostasis in $\mathrm{NaCl}$ stress environment. Plant Physiology, 109: 735- 742.

30. Poljakoff-mayber, A., G.F. Somers, E. Werker and J.I. Gallagher. 1994. Seeds of Kosteletzkya virginica (Malvaceace), their structure, germination and salt tolerance. American. Journal of Botany, 81: 54-59. 
31. Qureshi, A.S., M. Qadir, N. Heydari, H. Turral and A. Javadi. 2007. A review of management strategies for saltprone land and water resources in Iran. Colombo, Sri Lanka: International Water Management Institute. (IWMI Working Paper, 30: 125 pp (In Persian).

32. Ramagopal, S. 1990. Inhibition of seed germination by salt and its subsequent effect on embryonic protein synthesis in barley. Journal. Plant Physiology, 136: 621-625.

33. Ramazani, M., M. Taghvaei, M. Masoudi, A. Riahi and N. Behbahani. 2009. The evaluation of drought and salinity effects on germination and seedling growth caper (Capparis spinosa L.). Journal of Rangeland, 2: 411-420 (In Persian).

34. Redmann, R.E., M.Q. Q1 and M. Belyk. 1994. Growth of transgenic and standard canola (Brassica napus L.) varieties in response to soil salinity. Plant Science, 74: 797-799.

35. Rezaee, M. and T. Alinejad. 2004. Study of effect of salinity on germination of cumin. Proceedings of the First National Conference on Cumin, 84-85 (In Persian).

36. Salami, M.R., A. Safarnejad and H. Hamidi. 2006. Effect of salinity stress on morphological characters of Cuminum cyminum and Valeriana officinalis. Journal of Pajouh and Sazandegi, 72: 7783 (In Persian).

37. Soltani, A., M. Gholipoor and E. Zeinali. 2006. Seed reserve utilization and seedling growth of wheat as affected by drought and salinity. Environment. Exp. Botany, 55: 195-200 (In Persian).

38. Szaboles, I. 1994. Soils and salinization. In Handbook of plant and crop stress. CRC Edition, 2: 1-12.

39. Yazdani Biooki, R., P. Rezvani Moghaddam, H.R. Khazai and R. Ghorbani. 2010. Effect of drought and salinity stress in seed germination of Silybum marianum. Iranian Journal of Field Crops Research, 8: 12-19 (In Persian).

40. Zhu, J.K. 2001. Plant salt tolerance. Trends in Plant Science, 6: 66-71. 


\title{
The Effect of Drought and Salinity Stress on Seed Germination of (Alyssum Homalocarpum)
}

\author{
Ali Reza Ganjali ${ }^{1}$, Majed Ajorlo ${ }^{2}$ and Abbas Khaksafidi ${ }^{3}$ \\ 1- M.Sc. of Rangeland, University of Zabol (Correspondent Author: Gmail:reza.ganjalii@ gmail.com) \\ 2 and 3- Assistant Professor and Instructor. Universitv of Zabol \\ Received: April 4, $2015 \quad$ Accepted: February 16, 2016
}

\begin{abstract}
Due to the limited Alyssum habitats (Alyssum homalocarpum) in rangeland ecosystems, it is necessary to study various aspects of plant growth under different environmental conditions such as abiotic stresses. The aim of this study was to evaluate the effect of salinity and drought on Alyssum seed germination characteristics in laboratory conditions. The experiment was conducted based on completely randomized design with four replications in the fall of 2014 in the culturing and Proliferation laboratory of Zabol University. To create different levels of drought and salinity, polyethylene glycol 6000 and $\mathrm{NaCl}$ were used respectively .Drought treatments were included four levels (zero, $-0 / 25,-0 / 41,-0 / 99 \mathrm{MPa}$ ) and five levels of salinity (zero, 50,100,150, $200 \mathrm{mM}$ ). Variance analysis results showed that drought and salinity stress significantly affected Alyssum seed germination characteristics $(\mathrm{p}<0.05)$ Mean comparison showed that by increasing salinity and drought, germination percentage ,germination speed ,shoot length and root length and root and shoot dry weight decreased $(\mathrm{p}<0.05)$. The Alyssum seeds endured drought potential up to $-0 / 25 \mathrm{MPa}$ and the highest germination percentage was observed in this level however increasing drought causing reduced germination percentage. According to the higher seed germination rates in different levels of draught stress rather than salinity, it could be assumed that the sodium chloride solution has decreased germination more than the polyethylene glycol indicated the higher sensitivity of this species to salinity.
\end{abstract}

Keywords: Alyssum, Drought stress, Germination, Medicinal plant, Salinity stress 